\title{
CONCAVITY OF THE CONDITIONAL MEAN SOJOURN TIME IN THE PROCESSOR-SHARING QUEUE WITH BATCH ARRIVALS
}

\author{
JEONGSIM KIM
}

\begin{abstract}
For an $M / G / 1$ processor-sharing queue with batch arrivals, Avrachenkov et al. [1] conjectured that the conditional mean sojourn time is concave. However, Kim and Kim [5] showed that this conjecture is not true in general. In this paper, we show that this conjecture is true if the service times have a hyperexponential distribution.
\end{abstract}

\section{Introduction}

The processor-sharing queues have been of considerable interest and are useful in modeling some computer and communication systems. Under the processor-sharing service discipline, each customer receives an equal share of the processor, i.e., when $n$ customers are present in the system, each customer receives service at a rate $\frac{1}{n}$.

The processor-sharing discipline was introduced by Kleinrock [6] and has been studied for a few decades. Many researchers have investigated for various processor-sharing queues with single arrivals. However, relatively little discussion has been paid to the processor-sharing queue with batch arrivals in the literature. Batch arrivals are often used to model the burstiness in the arrival process. An application of the processor-sharing queue with batch arrivals can be found in Avrachenkov et al. [1].

Kleinrock et al. [7] first studied the $M / G / 1$ processor-sharing queue with batch arrivals and showed that the derivative of the mean sojourn time conditioned on the required service time (conditional mean sojourn time) satisfies an integral equation. This equation cannot be easily solved. They solved this equation for a special case of service time distributions, which is only slightly more general than exponential distributions. Bansal [2], using Kleinrock's integral equation, obtained the Laplace-Stieltjes transform of the conditional

Received April 19, 2009; Revised June 15, 2009.

2000 Mathematics Subject Classification. 60K25.

Key words and phrases. processor-sharing queue, batch arrivals, sojourn time, concavity.

This work was supported by the research grant of the Chungbuk National University in 2008 .

(C)2010 The Korean Mathematical Society 
mean sojourn time, when the service times of customers have a generalized hyperexponential distribution and more generally for distributions with rational Laplace transforms. He also obtained the unconditional mean sojourn time for both of those special cases. Feng and Misra [3] provided bounds for the conditional mean sojourn time. Avrachenkov et al. [1] proved the existence and uniqueness of a solution to Kleinrock's integral equation. Furthermore, they provided asymptotical analysis as well as tight bounds for the conditional and unconditional mean sojourn times. Rege and Sengupta [8] found the sojourn time distribution for a tagged customer, given the service times of all customers in the system. They also obtained the sojourn time distribution at an arrival instant for a customer whose service requirement is known, only in two special cases: one is when the service times have exponential distribution and the other is when the batch size is 1. Recently, Kim and Kim [4] obtained the moments of the queue-length and the sojourn time, when the service times have a phase-type distribution.

For the M/G/1 processor-sharing queue with batch arrivals, Avrachenkov et al. [1] conjectured that the conditional mean sojourn time is concave. However, Kim and Kim [5] showed that this conjecture is not true in general. In this paper, we show that this conjecture is true if the service times have a hyperexponential distribution.

\section{Main result}

We consider an M/HE/1 processor-sharing queueing system with batch arrivals. This kind of system is usually denoted by $M^{X} / \mathrm{HE} / 1$ PS queue. The customers arrive in batches of random size $B$. We assume that the batch size $B$ has a finite second moment. The batches arrive according to a Poisson process with rate $\lambda$. The service times have a hyperexponential distribution of order $K$ with the following distribution function:

$$
F(x)=1-\sum_{i=1}^{K} \alpha_{i} e^{-\mu_{i} x},
$$

where $\mu_{i}>0, \alpha_{i}>0$ for all $i$ and $\sum_{i=1}^{K} \alpha_{i}=1$. We note that the hyperexponential distribution of order $K$ consists of $K$ exponential "phases". Let $\boldsymbol{\alpha}=\left(\alpha_{1}, \ldots, \alpha_{K}\right)$. The offered load $\rho$ is $\rho \equiv \lambda \mathbb{E}(B) \sum_{i=1}^{K} \frac{\alpha_{i}}{\mu_{i}}$ and is assumed to be less than 1 . We also denote by $\rho_{i}, i=1, \ldots, K$, the offered load by phase $i$, i.e., $\rho_{i} \equiv \lambda \mathbb{E}(B) \frac{\alpha_{i}}{\mu_{i}}$.

Let us denote by $T(x)$ the conditional mean sojourn time for a customer with service time $x$. Our main result is the next theorem, which asserts that the conditional mean sojourn time $T(x)$ is a concave function, when the service times have a hyperexponential distribution. The proof is deferred to the next section. 
Theorem 1. We consider the $M^{X} / H E / 1$ PS queue with service time distribution as (1). Then the conditional mean sojourn time $T(x)$ satisfies $T^{\prime \prime}(x) \leq 0$. Hence $T(x)$ is concave in $x$.

As a corollary of the theorem above, we obtain a result on the slowdown, which is defined as the sojourn time divided by the service time. Let $S(x)$ be the slowdown of the customer with service time $x$.

Corollary 1. For the $M^{X} / H E / 1$ PS queue with service time distribution as (1), the mean slowdown $\mathbb{E}(S(x))$ is nonincreasing in $x$.

Proof. Since $\mathbb{E}(S(x))=\frac{T(x)}{x}$,

$$
\frac{d}{d x} \mathbb{E}(S(x))=\frac{T^{\prime}(x) x-T(x)}{x^{2}} .
$$

By Theorem 1, $T^{\prime}(x)$ is nonincreasing, and so

$$
T(x)=\int_{0}^{x} T^{\prime}(y) d y \geq \int_{0}^{x} T^{\prime}(x) d y=T^{\prime}(x) x .
$$

Therefore, $\frac{d}{d x} \mathbb{E}(S(x)) \leq 0$.

\section{Proof of main result}

In this section we prove Theorem 1. For the convenience of the reader we repeat an expression for the conditional mean sojourn time, together with its first and second derivatives, from Kim and Kim [5]. We also give an expression for the mean number of customers at steady state. Denote by $N_{i}, i=1, \ldots, K$, the number of customers in phase $i$ service, at steady state.

Proposition 1 ([5]). For the $M^{X} / H E / 1$ PS queue with service time distribution (1), $T(x), T^{\prime}(x)$ and $T^{\prime \prime}(x)$ are given as follows:

$$
\begin{aligned}
& T(x)= \frac{x}{1-\rho}+\left(\left(\mathbb{E}\left(N_{1}\right), \ldots, \mathbb{E}\left(N_{K}\right)\right)-\frac{1}{1-\rho}\left(\rho_{1}, \ldots, \rho_{K}\right)+\frac{\mathbb{E}(B(B-1))}{\mathbb{E}(B)} \boldsymbol{\alpha}\right) \\
& \times\left(\operatorname{diag}\left(\frac{1}{\mu_{1}}, \ldots, \frac{1}{\mu_{K}}\right)+\frac{1}{1-\rho}\left(\frac{1}{\mu_{1}}, \ldots, \frac{1}{\mu_{K}}\right)^{\top}\left(\rho_{1}, \ldots, \rho_{K}\right)\right)\left(\mathbf{1}-e^{\widetilde{A} x} \mathbf{1}\right), \\
& T^{\prime}(x)= \frac{1}{1-\rho}+\left(\left(\mathbb{E}\left(N_{1}\right), \ldots, \mathbb{E}\left(N_{K}\right)\right)-\frac{1}{1-\rho}\left(\rho_{1}, \ldots, \rho_{K}\right)+\frac{\mathbb{E}(B(B-1))}{\mathbb{E}(B)} \boldsymbol{\alpha}\right) e^{\widetilde{A} x} \mathbf{1}, \\
& \text { (2) } T^{\prime \prime}(x)=\left(\left(\mathbb{E}\left(N_{1}\right), \ldots, \mathbb{E}\left(N_{K}\right)\right)-\frac{1}{1-\rho}\left(\rho_{1}, \ldots, \rho_{K}\right)+\frac{\mathbb{E}(B(B-1))}{\mathbb{E}(B)} \boldsymbol{\alpha}\right) e^{\widetilde{A} x} \widetilde{A} \mathbf{1},
\end{aligned}
$$

where $\widetilde{A}=\lambda \mathbb{E}(B) \mathbf{1} \boldsymbol{\alpha}-\operatorname{diag}\left(\mu_{1}, \ldots, \mu_{K}\right)$.

Throughout the paper, 1 denotes a column vector with all its components equal to one, the superscript $T$ denotes the transpose of a vector or a matrix.

The mean number of customers $\left(\mathbb{E}\left(N_{1}\right), \ldots, \mathbb{E}\left(N_{K}\right)\right)$ is expressed as the following proposition, which is immediate from equations (6), (8) and (9) in Kim and $\operatorname{Kim}[4]$. 
Proposition 2. For the $M^{X} / H E / 1$ PS queue with service time distribution (1), we have

$$
\left(\mathbb{E}\left(N_{1}\right), \ldots, \mathbb{E}\left(N_{K}\right)\right)=\mathbf{1}^{\top} \widetilde{R}+\left(\rho_{1}, \ldots, \rho_{K}\right),
$$

where $\widetilde{R}$ is the solution of

$$
\begin{aligned}
\widetilde{A}^{\top} \widetilde{R}+\widetilde{R} \widetilde{A}= & -\lambda \mathbb{E}(B)\left(\boldsymbol{\alpha}^{\top}\left(\rho_{1}, \ldots, \rho_{K}\right)+\left(\rho_{1}, \ldots, \rho_{K}\right)^{\top} \boldsymbol{\alpha}\right) \\
& -\lambda \mathbb{E}(B(B-1)) \boldsymbol{\alpha}^{\top} \boldsymbol{\alpha}
\end{aligned}
$$

with $\widetilde{A}$ given in Proposition 1.

Remark. It is known that (3) has a unique solution for $\widetilde{R}$ (See Kim and Kim [4]).

The following lemma provides an expression for $\left(\mathbb{E}\left(N_{1}\right), \ldots, \mathbb{E}\left(N_{K}\right)\right)$ in the case when $\boldsymbol{\alpha}=\left(\frac{1}{K}, \ldots, \frac{1}{K}\right)$, i.e., $\boldsymbol{\alpha}=\frac{1}{K} \mathbf{1}^{\top}$.

Lemma 1. If $\boldsymbol{\alpha}=\frac{1}{K} \mathbf{1}^{\top}$, then there are $c_{n} \geq 0, n=0,1,2 \ldots$, such that

$$
\left(\mathbb{E}\left(N_{1}\right), \ldots, \mathbb{E}\left(N_{K}\right)\right)=\frac{1}{1-\rho}\left(\rho_{1}, \ldots, \rho_{K}\right)+\sum_{n=0}^{\infty} c_{n} \mathbf{1}^{\top} C^{n},
$$

where $C=\frac{\lambda \mathbb{E}(B)}{K} \mathbf{1 1}^{\top}-\operatorname{diag}\left(\mu_{1}, \ldots, \mu_{K}\right)+\mu^{*} I$ with $\mu^{*}=\max \left\{\mu_{1}, \ldots, \mu_{K}\right\}$.

Proof. According to Proposition 2, we have

$$
\left(\mathbb{E}\left(N_{1}\right), \ldots, \mathbb{E}\left(N_{K}\right)\right)=\mathbf{1}^{\top} R+\left(\rho_{1}, \ldots, \rho_{K}\right),
$$

where $R$ is the solution of

$$
\begin{aligned}
A R+R A= & -\frac{\lambda \mathbb{E}(B)}{K}\left(\mathbf{1}\left(\rho_{1}, \ldots, \rho_{K}\right)+\left(\rho_{1}, \ldots, \rho_{K}\right)^{\top} \mathbf{1}^{\top}\right) \\
& -\frac{\lambda \mathbb{E}(B(B-1))}{K^{2}} \mathbf{1 1}^{\top},
\end{aligned}
$$

with $A=\frac{\lambda \mathbb{E}(B)}{K} \mathbf{1 1} \mathbf{1}^{\top}-\operatorname{diag}\left(\mu_{1}, \ldots, \mu_{K}\right)$. Note that the matrix $A$ is symmetric. The matrix $R$ can be decomposed as

$$
R=U+V,
$$

where $U$ and $V$ are solutions of

$$
\begin{aligned}
& A U+U A=-\frac{\lambda \mathbb{E}(B)}{K}\left(\mathbf{1}\left(\rho_{1}, \ldots, \rho_{K}\right)+\left(\rho_{1}, \ldots, \rho_{K}\right)^{\top} \mathbf{1}^{\top}\right), \\
& A V+V A=-\frac{\lambda \mathbb{E}(B(B-1))}{K^{2}} \mathbf{1 1}^{\top},
\end{aligned}
$$

respectively. Thus

$$
\left(\mathbb{E}\left(N_{1}\right), \ldots, \mathbb{E}\left(N_{K}\right)\right)=\mathbf{1}^{\top} U+\mathbf{1}^{\top} V+\left(\rho_{1}, \ldots, \rho_{K}\right) .
$$

For a moment, we consider an $\mathrm{M} / \mathrm{HE} / 1$ PS queue with arrival rate $\lambda \mathbb{E}(B)$ and service time distribution $F(x)=1-\sum_{i=1}^{K} \frac{1}{K} e^{-\mu_{i} x}$. Let $\mathbb{E}\left(N_{i}^{\text {single }}\right), i=$ $1, \ldots, K$, denote the mean number of customers in phase $i$ service for this 
queueing system, at steady state. Then, by the equations (6) and (9) in Kim and $\operatorname{Kim}[4]$,

$$
\left(\mathbb{E}\left(N_{1}^{\text {single }}\right), \ldots, \mathbb{E}\left(N_{K}^{\text {single }}\right)\right)=\mathbf{1}^{\top} U+\left(\rho_{1}, \ldots, \rho_{K}\right) .
$$

It is well-known that

$$
\left(\mathbb{E}\left(N_{1}^{\text {single }}\right), \ldots, \mathbb{E}\left(N_{K}^{\text {single }}\right)\right)=\frac{1}{1-\rho}\left(\rho_{1}, \ldots, \rho_{K}\right) .
$$

Therefore

$$
\mathbf{1}^{\top} U+\left(\rho_{1}, \ldots, \rho_{K}\right)=\frac{1}{1-\rho}\left(\rho_{1}, \ldots, \rho_{K}\right) .
$$

Substituting the above into (5) leads to

$$
\left(\mathbb{E}\left(N_{1}\right), \ldots, \mathbb{E}\left(N_{K}\right)\right)=\frac{1}{1-\rho}\left(\rho_{1}, \ldots, \rho_{K}\right)+\mathbf{1}^{\top} V .
$$

Now we show that, for each $n=0,1, \ldots$, there are $c_{n i}$ such that

$$
\mathbf{1}^{\top} C^{n} V=\sum_{i=0}^{\infty} c_{n i} \mathbf{1}^{\top} C^{i}
$$

Since $A=C-\mu^{*} I,(4)$ becomes

$$
V=\frac{1}{2 \mu^{*}}\left(C V+V C+\frac{\lambda \mathbb{E}(B(B-1))}{K^{2}} \mathbf{1 1}^{\top}\right) .
$$

Define $V^{(j)}, j=0,1, \ldots$, as

$$
\begin{aligned}
V^{(0)} & =O \\
V^{(j+1)} & =\frac{1}{2 \mu^{*}}\left(C V^{(j)}+V^{(j)} C+\frac{\lambda \mathbb{E}(B(B-1))}{K^{2}} \mathbf{1 1}^{\top}\right), j=0,1, \ldots
\end{aligned}
$$

By induction on $j$, it can be shown that for every $n=0,1, \ldots$, there are $c_{n i}^{(j)}$ such that

$$
\mathbf{1}^{\top} C^{n} V^{(j)}=\sum_{i=0}^{\infty} c_{n i}^{(j)} \mathbf{1}^{\top} C^{i} .
$$

Furthermore, $c_{n i}^{(j)}$ can be chosen so that $c_{n i}^{(j)}$ is nondecreasing in $j$. Let

$$
\lim _{j \rightarrow \infty} c_{n i}^{(j)}=c_{n i}
$$

Since $V^{(j)}$ converges to $V$, the assertion (7) follows. Finally, substituting the special case $n=0$ of (7) into (6) completes the proof.

$$
\begin{aligned}
\text { If } \boldsymbol{\alpha} & =\frac{1}{K} \mathbf{1}^{\top}, \text { then, by }(2), \\
T^{\prime \prime}(x) & =\left(\left(\mathbb{E}\left(N_{1}\right), \ldots, \mathbb{E}\left(N_{K}\right)\right)-\frac{1}{1-\rho}\left(\rho_{1}, \ldots, \rho_{K}\right)+\frac{\mathbb{E}(B(B-1))}{K \mathbb{E}(B)} \mathbf{1}^{\top}\right) e^{A x} A \mathbf{1} .
\end{aligned}
$$


Therefore, by Lemma 1, we have

$$
T^{\prime \prime}(x)=\sum_{n=0}^{\infty} c_{n} \mathbf{1}^{\top} C^{n} e^{A x} A \mathbf{1}+\frac{\mathbb{E}(B(B-1))}{K \mathbb{E}(B)} \mathbf{1}^{\top} e^{A x} A \mathbf{1} .
$$

Finally, to prove Theorem 1, we need one more lemma.

Lemma 2. If $\boldsymbol{\alpha}=\frac{1}{K} \mathbf{1}^{\top}$, then $\mathbf{1}^{\top} C^{n} e^{A x} A \mathbf{1} \leq 0$ for all $x \geq 0$ and $n=$ $0,1, \ldots$, where $A=\frac{\lambda \mathbb{E}(B)}{K} \mathbf{1 1}^{\top}-\operatorname{diag}\left(\mu_{1}, \ldots, \mu_{K}\right)$ and $C=A+\mu^{*} I$ with $\mu^{*}=$ $\max \left\{\mu_{1}, \ldots, \mu_{K}\right\}$.

Proof. Note that the matrix $C$ is symmetric. Thus $C$ can be decomposed as

$$
C=P^{\top} \operatorname{diag}\left(\sigma_{1}, \ldots, \sigma_{K}\right) P,
$$

where $P$ is an orthogonal matrix and $\sigma_{1}, \ldots, \sigma_{K}$ are eigenvalues of $C$. Since $A=C-\mu^{*} I$, we have

$$
A=P^{\top} \operatorname{diag}\left(\sigma_{1}-\mu^{*}, \ldots, \sigma_{K}-\mu^{*}\right) P,
$$

$\mathrm{SO}$

$$
e^{A x}=P^{\top} \operatorname{diag}\left(e^{\left(\sigma_{1}-\mu^{*}\right) x}, \ldots, e^{\left(\sigma_{K}-\mu^{*}\right) x}\right) P .
$$

Therefore

$$
\begin{aligned}
& \mathbf{1}^{\top} C^{n} e^{A x} A \mathbf{1} \\
= & \mathbf{1}^{\top} P^{\top} \operatorname{diag}\left(\sigma_{1}^{n} e^{\left(\sigma_{1}-\mu^{*}\right) x}\left(\sigma_{1}-\mu^{*}\right), \ldots, \sigma_{K}^{n} e^{\left(\sigma_{K}-\mu^{*}\right) x}\left(\sigma_{K}-\mu^{*}\right)\right) P \mathbf{1} \\
= & \sum_{i=1}^{K} \sigma_{i}^{n} e^{\left(\sigma_{i}-\mu^{*}\right) x}\left(\sigma_{i}-\mu^{*}\right)\left((P \mathbf{1})_{i}\right)^{2},
\end{aligned}
$$

where $(\boldsymbol{w})_{i}$ indicates the $i$ th component of a vector $\boldsymbol{w}$.

For any real column vector $\boldsymbol{\xi}=\left(\xi_{1}, \ldots, \xi_{K}\right)^{\top}$,

$$
\begin{aligned}
\boldsymbol{\xi}^{\top} C \boldsymbol{\xi} & =\frac{\lambda \mathbb{E}(B)}{K} \boldsymbol{\xi}^{\top} \mathbf{1} \mathbf{1}^{\top} \boldsymbol{\xi}+\boldsymbol{\xi}^{\top} \operatorname{diag}\left(\mu^{*}-\mu_{1}, \ldots, \mu^{*}-\mu_{K}\right) \boldsymbol{\xi} \\
& =\frac{\lambda \mathbb{E}(B)}{K}\left(\boldsymbol{\xi}^{\top} \mathbf{1}\right)^{2}+\sum_{i=1}^{K} \xi_{i}^{2}\left(\mu^{*}-\mu_{i}\right) \\
& \geq 0 .
\end{aligned}
$$

Therefore $C$ is nonnegative definite, which implies that $\sigma_{i}, i=1, \ldots, K$, are all nonnegative. Furthermore, $\sigma_{i}<\mu^{*}$ for all $i=1, \ldots, K$, because $C$ is a positive matrix and

$$
\left(\frac{1}{\mu_{1}}, \ldots, \frac{1}{\mu_{K}}\right) C=\mu^{*}\left(\frac{1}{\mu_{1}}, \ldots, \frac{1}{\mu_{K}}\right)-(1-\rho) \mathbf{1}^{\top}<\mu^{*}\left(\frac{1}{\mu_{1}}, \ldots, \frac{1}{\mu_{K}}\right) .
$$

Thus we have

$$
0 \leq \sigma_{i}<\mu^{*}, i=1, \ldots, K,
$$

which, together with (9), completes the proof. 
Now we are ready to prove Theorem 1 . We break up the proof into 3 steps.

Proof of Theorem 1.

STEP 1. The case that $\boldsymbol{\alpha}=\frac{1}{K} \mathbf{1}^{\top}$ :

By (8), together with Lemma 2 , we have $T^{\prime \prime}(x) \leq 0$.

STEP 2. The case that $\alpha_{1}, \ldots, \alpha_{K}$ are rational numbers:

The rational numbers $\alpha_{1}, \ldots, \alpha_{K}$ are written as

$$
\alpha_{1}=\frac{p_{1}}{r}, \ldots, \alpha_{K}=\frac{p_{K}}{r},
$$

where $p_{1}, \ldots, p_{K}$ and $r$ are positive integers. Note that $p_{1}+\cdots+p_{K}=r$. We observe that

$$
F(x)=1-\sum_{j=1}^{r} \widetilde{\alpha}_{j} e^{-\widetilde{\mu}_{j} x},
$$

where $\widetilde{\alpha}_{j}=\frac{1}{r}, j=1, \ldots, r$, and

$$
\widetilde{\mu}_{j}=\left\{\begin{array}{cl}
\mu_{1} & \text { if } 1 \leq j \leq p_{1} \\
\mu_{2} & \text { if } p_{1}+1 \leq j \leq \sum_{i=1}^{2} p_{i} \\
\vdots & \\
\mu_{K} & \text { if } \sum_{i=1}^{K-1} p_{i}+1 \leq j \leq \sum_{i=1}^{K} p_{i} .
\end{array}\right.
$$

Therefore the result follows from Step 1.

STEP 3. The case that $\alpha_{1}, \ldots, \alpha_{K}$ are positive real numbers: Choose positive rational numbers $\alpha_{1}^{(n)}, \ldots, \alpha_{K}^{(n)}, n=1,2, \ldots$, such that

$$
\sum_{i=1}^{K} \alpha_{i}^{(n)}=1, \lambda \mathbb{E}(B) \sum_{i=1}^{K} \frac{\alpha_{i}^{(n)}}{\mu_{i}}<1,
$$

and $\lim _{n \rightarrow \infty} \alpha_{1}^{(n)}=\alpha_{1}, \ldots, \lim _{n \rightarrow \infty} \alpha_{K}^{(n)}=\alpha_{K}$. For each $n$, consider the $M^{X} / \mathrm{HE} / 1$ PS queue where the service times have a hyperexponential distribution

$$
F^{(n)}(x)=1-\sum_{i=1}^{K} \alpha_{i}^{(n)} e^{-\mu_{i} x} .
$$

For this $M^{X} / \mathrm{HE} / 1 \mathrm{PS}$ queue with service time distribution $(10)$, let $T^{(n)}(x)$ be the conditional mean sojourn time for a customer with service time $x$ and $N_{i}^{(n)}, i=1, \ldots, K$, the number of customers in phase $i$ service, at steady state. Then we have that by Step 2,

$$
\left(T^{(n)}\right)^{\prime \prime}(x) \leq 0
$$


and by (2),

(12)

$$
\begin{aligned}
& \left(T^{(n)}\right)^{\prime \prime}(x) \\
= & \left(\left(\mathbb{E}\left(N_{1}^{(n)}\right), \ldots, \mathbb{E}\left(N_{K}^{(n)}\right)\right)-\frac{1}{1-\rho^{(n)}}\left(\rho_{1}^{(n)}, \ldots, \rho_{K}^{(n)}\right)+\frac{\mathbb{E}(B(B-1))}{\mathbb{E}(B)} \boldsymbol{\alpha}^{(n)}\right) e^{\widetilde{A}^{(n)} x} \widetilde{A}^{(n)} \mathbf{1},
\end{aligned}
$$

where

$$
\begin{aligned}
\rho_{i}^{(n)} & =\lambda \mathbb{E}(B) \frac{\alpha_{i}^{(n)}}{\mu_{i}}, i=1, \ldots, K, \\
\rho^{(n)} & =\sum_{i=1}^{K} \rho_{i}^{(n)}, \\
\boldsymbol{\alpha}^{(n)} & =\left(\alpha_{1}^{(n)}, \ldots, \alpha_{K}^{(n)}\right), \\
\widetilde{A}^{(n)} & =\lambda \mathbb{E}(B) \mathbf{1} \boldsymbol{\alpha}^{(n)}-\operatorname{diag}\left(\mu_{1}, \ldots, \mu_{K}\right) .
\end{aligned}
$$

We observe that $\lim _{n \rightarrow \infty} \rho_{i}^{(n)}=\rho_{i}, i=1, \ldots, K, \lim _{n \rightarrow \infty} \rho^{(n)}=\rho, \lim _{n \rightarrow \infty} \boldsymbol{\alpha}^{(n)}$ $=\boldsymbol{\alpha}$, and $\lim _{n \rightarrow \infty} \widetilde{A}^{(n)}=\widetilde{A}$. Furthermore, Proposition 2 implies that

$$
\lim _{n \rightarrow \infty} \mathbb{E} N_{i}^{(n)}=\mathbb{E} N_{i}, i=1, \ldots, K .
$$

Thus the right-hand side of (12) goes to the right-hand side of (2) as $n \rightarrow$ $\infty$. Therefore, by $(11)$, we have $T^{\prime \prime}(x) \leq 0$, which completes the proof of Theorem 1.

\section{References}

[1] K. Avrachenkov, U. Ayesta, and P. Brown, Batch arrival processor-sharing with application to multi-level processor-sharing scheduling, Queueing Syst. 50 (2005), no. 4, 459-480.

[2] N. Bansal, Analysis of the $M / G / 1$ processor-sharing queue with bulk arrivals, Oper. Res. Lett. 31 (2003), no. 5, 401-405.

[3] H. Feng and V. Misra, Asymptotic bounds for $M^{X} / G / 1$ processor sharing queues, Tech. Rep., CUCS-006-04, Columbia University, 2003.

[4] J. Kim and B. Kim, The processor-sharing queue with bulk arrivals and phase-type services, Perform. Eval. 64 (2007), no. 4, 277-297.

[5] _ Concavity of the conditional mean sojourn time in the $M / G / 1$ processor-sharing queue with batch arrivals, Queueing Syst. 58 (2008), no. 1, 57-64.

[6] L. Kleinrock, Time-shared systems: A theoretical treatment, J. Assoc. Comput. Mach. 14 (1967), 242-261.

[7] L. Kleinrock, R. R. Muntz, and E. Rodemich, The processor-sharing queueing model for time-shared systems with bulk arrivals, Networks 1 (1971), no. 1, 1-13.

[8] K. M. Rege and B. Sengupta, The $M / G / 1$ processor-sharing queue with bulk arrivals, in: Modelling and Performance Evaluation of ATM Technology, Elsevier, Amsterdam, 1993, 417-432.

Department of Mathematics Education

Chungbuk National University

Chungbuk 361-763, Korea

E-mail address: jeongsimkim@chungbuk.ac.kr 